\title{
Die strategische Wahl der Gewerkschaften - Erneuerung durch Organizing?
}

Vor dem Hintergrund einer tiefen Krise gewerkschaftlicher Repräsentation fragt der Beitrag nach Ansatzpunkten einer Erneuerung von Organisationsmacht. Gewerkschaften werden als Akteure betrachtet, die eine strategische Wahl bei der Erschließung neuer Machtressourcen besitzen. Organizing-Konzepte, wie sie inzwischen auch hierzulande erprobt werden, sind kein Patentrezept zur Überwindung der Mitgliederkrise. Den deutschen Gewerkschaften können sie aber wichtige Anregungen für eine strategische Neuausrichtung bieten.

\section{Einleitung}

Schwindende gewerkschaftliche Organisationsmacht wird in den Sozialwissenschaften seit Langem thematisiert (Dribbusch 2003). Allein zwischen 1993 und 2003 haben die europäischen Gewerkschaften im Durchschnitt $15 \%$ ihrer Mitglieder verloren. Die DGB-Gewerkschaften sind hier keine Ausnahme. Gegenwärtig entspricht ihre absolute Mitgliederzahl in Gesamtdeutschland nur noch jener der West-Gewerkschaften von 1969/70. Trotz aktuell positiver Gegentendenzen vor allem bei jüngeren Beschäftigten, drohen Mitgliederschwund, knappe Ressourcen und eine abnehmende Zahl von Aktiven selbst in einstigen Hochburgen gewerkschaftlicher Organisationsmacht in einen Ohnmachtszirkel gewerkschaftlicher Desorganisation umzuschlagen (Dörre/Röttger 2006, S. 227 268). Die tiefe Repräsentationskrise vor Augen, beschäftigen sich die „Labour Revitalization Studies" (Frege/Kelly 2004) mit Potenzialen gewerkschaftlicher Erneuerung. In Kontrast zum fatalistischen Tenor vieler Analysen lenken sie das Augenmerk auf die strategische Wahl, auf die Möglichkeiten von Gewerkschaften, kreativ zu handeln und sich selbst zu erneuern.

Lange Zeit eher mitleidig beäugt, gelten nun ausgerechnet einige US-Gewerkschaften als lebendiger Beweis für Alternativen zu bürokratisch verwaltetem Niedergang (Geiselberger 2007, S. 79f.). Und in der Tat, die Botschaften aus Übersee finden inzwischen auch hierzulande Gehör. Unter jüngeren Gewerkschaftern und Wissenschaftlern scheint ein regelrechter OrganizingHype ausgebrochen sein. Aber handelt es sich dabei um mehr als um eine jener
Moden, die nach ersten Misserfolgen genau so rasch verschwinden wie sie gekommen sind? Unsere These lautet: Ansätze, die unter Begriffen wie Organizing, Social Movement Unionism und Campaigning diskutiert werden, sind kein Universalschlüssel zur Behebung der Mitgliederkrise; gleichwohl können sie auch den deutschen Gewerkschaften wichtige Anregungen zur Erneuerung ihrer Organisationsmacht liefern. ${ }^{1}$

\section{Machtressourcen und Gewerkschaften}

Gewerkschaftsmacht basiert auf dem Bemühen von Lohnabhängigen, ihre Konkurrenzen zumindest zeitweilig und in den Grenzen bestimmter Branchen und Territorien zu überwinden, um auf der Basis geteilter Interessen und Wertorientierungen gemeinsame Ziele zu verfolgen. Insofern handelt es sich um einen Spezialfall von Arbeiter- oder besser: von Lohnabhängigenmacht. Im Anschluss an Wright (2000, S. 962) und Silver (2005, S. 30-44) kann zwischen struktureller und Organisationsmacht unterschieden werden. Strukturelle Macht erwächst aus der Stellung spezifischer Arbeiter- und Angestelltengruppen im ökonomischen System. Sie kann sich in primärer Verhandlungsmacht, die auf einer angespannten Arbeitsmarktsituation beruht, ebenso ausprägen wie in Produktionsmacht, die sich über eine besondere strategische Stellung in Arbeitsprozessen konstituiert. Davon zu unterscheiden ist Organisationsmacht, die erst aus dem $\mathrm{Zu}$ sammenschluss zu kollektiven politischen oder gewerkschaftlichen Organisationen entsteht. Organisationsmacht kann struk- turelle Verhandlungs- und Produktionsmacht teilweise substituieren, jedoch nicht vollständig ersetzen. Strukturelle Macht wird häufig spontan ausgeübt. Sie tritt in Gestalt von „labour unrest“ (Silver 2005, S. 11, 44ff.), plötzlichen Unruhen und situativer Empörung ebenso auf wie als informelle Sabotage oder Absentismus in Produktionsprozessen. Organisationsmacht ist demgegenüber prinzipiell auf handlungsfähige Gewerkschaften, Parteien oder ähnliche Akteure angewiesen.

Neben diesen beiden Machtressourcen existiert eine dritte Quelle, die institutionelle Macht. Ihre Besonderheit ergibt sich aus dem Faktum, dass Institutionen soziale Kompromisse über ökonomische Konjunkturen und kurzzeitige Veränderungen gesellschaftlicher Kräfteverhältnisse hinweg festschreiben und teilweise gesetzlich fixieren. Auf diese Weise wird Arbeitermacht in gesellschaftliche Institutionen inkorporiert (Fligstein 2001). Institutionalisierte Arbeitsbeziehungen legen den Gewerkschaften Handlungsstrategien nahe, die auch dann noch überzeugend erscheinen, wenn sich gesellschaftliche Kräftever-

\footnotetext{
1 Der Artikel basiert auf einer Studie der AG Strategic Unionism (Brinkmann et al. 2008). Zur Arbeitsgruppe gehören Ulrich Brinkmann, Hae-Lin Choi, Richard Detje, Hajo Holst, Serhat Karakayali und Catharina Schmalstieg.
}

Klaus Dörre, Dr., Prof. für Arbeits-, Industrieund Wirtschaftssoziologie an der FriedrichSchiller-Universität Jena. Arbeitsschwerpunkte: Prekäre Beschäftigung, Strategic Unionism, Kapitalismustheorien. e-mail: klaus.doerre@uni-jena.de 
hältnisse bereits gravierend verändert haben. Die Nutzung institutioneller Macht setzt in einem solchen Fall voraus, dass die Gewerkschaften trotz nachlassender Bindungsfähigkeit gesellschaftlich weiter als authentische Repräsentanten der Lohnabhängigen anerkannt werden. Die Chance, institutionelle Macht über ihre Erzeugungsbedingungen hinaus wirksam werden zu lassen, kann Gewerkschaften dazu animieren, Repräsentationsdefizite durch institutionenkonformes Verhalten kompensieren zu wollen. Dabei laufen die Lohnabhängigenorganisationen jedoch beständig Gefahr, Handlungsstrategien zu konservieren, denen die Geschäftsgrundlage allmählich abhanden kommt.

\section{Erosion institutioneller Macht}

Die Nachkriegsjahrzehnte lassen sich in Deutschland - wie auch in anderen kontinentaleuropäischen kapitalistischen Systemen - als eine Ära des Ausbaus institutioneller Gewerkschaftsmacht beschreiben. Wie nie zuvor in der Geschichte ist Lohnarbeit mit einem Sozialeigentum zur Existenz- und Statussicherung verkoppelt worden, das sich in garantierten Rentenansprüchen, Kündigungs- und Arbeitsschutz, Mitbestimmungsrechten sowie verbindlichen tariflichen Normen manifestierte. Die Koppelung von Lohnarbeit mit starken Schutz- und Partizipationsrechten konstituierte einen Bürgerstatus, der zuvor besitzlosen Klassen trotz fortbestehender Ungleichheiten zu einem respektierten Status in der Gesellschaft verhalf. Die Gewerkschaften betätigten sich als eine treibende Kraft dieser Entwicklung. Je erfolgreicher sie in ihrem Bestreben waren, abhängig Beschäftigte am Produktivitätsfortschritt zu beteiligen und sie mit kollektiven Partizipations- und Schutzrechten auszustatten, desto stärker veränderten sie sich selbst. In den wohlfahrtsstaatlich regulierten kapitalistischen Systemen verschob sich das Zentrum ihres strategischen Handelns von struktureller und Organisationsmacht hin zu institutioneller Macht. Für Deutschland bedeutete dies, dass die Gewerkschaften und die mit ihnen verbündeten Betriebsräte die „intermediäre“ Logik des dualen Systems der Interessenrepräsentation, die spezifische Kombination von Tarifautono- mie und betrieblicher Mitbestimmung, möglichst effizient zu nutzen suchten. Auf diese Weise gelang es, institutionelle Gewerkschaftsmacht immer stärker auszubauen.

Spätestens seit Mitte der 1990er Jahre erleben wir eine gewisse Umkehrung dieser Entwicklung. Eine neue „kapitalistische Landnahme" zielt auf die politischen Beschränkungen und regulierende Strukturen, in die das Marktgeschehen über Jahrzehnte hinweg eingebettet war (Harvey 2006). In Deutschland vollzieht sich die Schwächung institutioneller Arbeitermacht nicht in Gestalt einer neoliberalen Rosskur. Sie verläuft eher schleichend und im Rahmen scheinbar intakter, noch weitgehend stabiler Institutionen. Gerade an der Nahtstelle von Tarifautonomie und betrieblicher Mitbestimmung macht sie sich gleichwohl massiv bemerkbar. Seit Jahren werden Tarifabschlüsse selbst in großen Unternehmen durch eine zweite, betriebs- und unternehmensbezogene Verhandlungsrunde korrigiert, die - häufig im Gegenzug für befristete Beschäftigungsgarantien - Abweichungen bei Entlohnung, Arbeitszeiten und Arbeitsbedingungen in dezentralen Wettbewerbspakten festschreiben (Huber et al. 2006). Betrieblichen Konzessionspolitiken und Verhandlungen, bei denen es im Grunde um das Ausmaß geht, in welchem Lohnarbeit an Marktrisiken rückgebunden wird, signalisieren offenbar einen Umschlagpunkt im Verhältnis von institutioneller und Organisationsmacht der Gewerkschaften. Anhaltender Druck auf die Institution Flächentarifvertrag ${ }^{2}$ verbindet sich mit der - seit Langem akuten und nun vor allem im Osten dramatisch zugespitzten - Mitgliederkrise. Pointiert formuliert: Die gewerkschaftliche Organisationsmacht reicht nicht mehr aus, um die Chancen, die das Institutionensystem bietet, interessenpolitisch zu nutzen. Zwar vollzieht sich dieser Prozess ungleichzeitig, nach Branchen differenziert und keineswegs linear; es gibt sogar Anhaltspunkte einer Re-Institutionalisierung von Lohnabhängigenmacht (Tarife im Reinigungsgewerbe, Mindestlöhne im Postgewerbe). Auch lässt sich darüber streiten, ob es sich noch immer um eine kontrollierte Dezentralisierung oder bereits um die Symptome einer Systemkrise korporativer Arbeitsbeziehungen handelt. In jedem Fall werden sich die Gewerkschaften künftig aber immer weniger darauf verlassen können, dass ihre Position in eingespielten Verhandlungsprozeduren auch dann erhalten bleibt, wenn ihre Organisationsmacht kontinuierlich schwächer wird.

\section{Organizing - strategischer Organisationswandel}

Nun darf die Erosion institutioneller Macht, wie sie sich in vielen Metropolenkapitalismen abzeichnet, nicht mit einem generellen Verschwinden von Arbeitermacht gleichgesetzt werden. Beverly Silver (2005) hat in ihrer eindrucksvollen Studie gezeigt, wie geografische Verlagerungen von Produktionsstandorten neue raumzeitliche „Fixierungen“" von Kapital, und mit ihnen neue Arbeiterklassen und Arbeiterbewegungen an den jeweils bevorzugten Produktionsstandorten, hervorbringen. Die Autorin skizziert eine räumliche Diffusion von Produktionsmacht, welche sich in vielen Ländern des Südens zunächst in „labour unrest", in spontanen Unruhen und Aufständen, äußert. Es finden sich aber auch Beispiele für eine Revitalisierung gewerkschaftlicher Organisationsmacht - und das nicht nur in sich entwickelnden Ländern wie Brasilien, Südkorea oder Südafrika, sondern auch in Zentrumsstaaten wie den USA.

Stilbildend für die internationale Debatte um gewerkschaftliche Erneuerung ist das Beispiel der Service Employees International Union (SEIU) und ihrer inzwischen legendären Kampagne „Justice for Janitors" (vgl. Beitrag von Choi in diesem Heft). Die SEIU hat sich infolge eines umfassenden Organisationswandels innerhalb kurzer Zeit von einer dahinsiechenden Business Union in eine konfliktfähige Gewerkschaft mit den höchsten Mitgliederzuwächsen in den USA gemausert. Andere nordamerikanische Gewerkschaften wie UNITE, HERE, UBC und LIUNA ${ }^{3}$ haben

\footnotetext{
2 Z. B. infolge von Mitgliedschaften ohne Tarifbindung in einigen Arbeitgeberverbänden, aufgrund von Tarifdumping, der Tarifierung von Niedriglöhnen und tariffreier Zonen in einzelnen Branchen und Unternehmen.

3 UNITE: Union of Needletrades, Industrial and Textile Employees, HERE: Hotel Employees and Restaurant Employees International Union, UBC: United Brotherhood of Carpenters and Joiners of America, LIUNA: Laborers International Union of North America.
} 
sich inzwischen ebenfalls für eine Ausrichtung am sogenannten „Organizing-Modell“ entschieden. ${ }^{4}$ Markenzeichen der genannten Gewerkschaften ist, dass sie sämtliche Aktivitäten mit dem Ziel einer nachhaltigen Stärkung ihrer Organisationsmacht verbinden. Und just diese Fokussierung dürfte wohl erheblich zur raschen internationalen Rezeption des „Organizing-Modells" beigetragen haben.

Die Redeweise von einem "Modell“ suggeriert indessen eine begrifflich-konzeptionelle Eindeutigkeit, die so nicht existiert. Gegenwärtig wird unter gewerkschaftlichen Praktikern und Wissenschaftlern kontrovers diskutiert, welche Handlungsstrategien, Organisationsformen und interessenpolitischen Inhalte mit dieser Organizing-Kategorie bezeichnet werden können. In der deutschen Debatte werden teilweise Definitionen favorisiert, die sich wesentlich auf Organisationstechniken und Methoden der Mitgliederwerbung konzentrieren (z. B. Dribbusch 2007, S. 30ff.). Das ist durchaus legitim, zumal Fragen nach der Übertragbarkeit von Organizing-Ansätzen auf deutsche Verhältnisse sich auf diese Weise pragmatisch beantworten lassen. Allerdings koppelt ein solch enges Verständnis die Organizing-Debatte von der inhaltlichen Dimension gewerkschaftlicher Erneuerung ab. Um dies zu vermeiden, orientieren wir uns an einem weiten, eher analytischen Organizing-Verständnis, wie es u. a. Voss/Sherman (2000) vorgeschlagen haben. In ihrem Klassiker der Labour Revitalization Studies (LBS)-Literatur zeichnen sie nach, wie bürokratische Strukturen und eingeschliffene Praktiken so verändert werden können, dass es zu einer Wiederbelebung gewerkschaftlicher Aktivitäten und zu einer erhöhten Mitgliederbindung kommt. Entscheidend ist demnach der Wechsel von einem Service-Modell, das eine weitgehend passive Mitgliedschaft über qualifizierte Dienstleistungen zu binden sucht, zu einem Organizing-Modell, das auf Mitgliedermobilisierung setzt, die Arbeitspraxis der Basisorganisationen verändert und neue, partizipative Organisationsstrukturen schafft.

Von Beispielen aus den USA inspiriert, arbeiten Voss/Sherman (2000) drei Faktorenbündel heraus, die den erfolgreichen Organisationswandel erklären sollen. Zwei Beobachtungen beziehen sich auf innerorganisatorische Phänomene. Zum einen schafft das verbreitete Bewusstsein über eine tiefe politische Krise der Organisation an der Basis Voraussetzungen für einen Führungswechsel. Das neue Personal, das sich lokal durchsetzt, verbindet die eigene Positionierung mit einem Strategiewechsel. Zum anderen bleibt der Wandel aber nicht auf die örtlichen Gewerkschaftsgliederungen beschränkt. Er erfasst die gesamte nationale Organisation und ist, wie im Fall der SEIU, teilweise mit heftigen Konflikten an der Spitze der Gewerkschaft verbunden. Von zentraler Bedeutung ist indessen eine dritte Beobachtung: Ein Teil der Führungskräfte, die - mitunter aufgrund von Druck aus der Zentrale - neu eingestellt werden, kommt „von außen“ und verfügt über Erfahrungen mit sozialen Bewegungen, Graswurzelinitiativen und Stadtteilarbeit. Den bewegungssozialisierten Gewerkschaftern fällt es offenbar leichter, einen umfassenden Strategiewechsel in Gang zu setzen. Sie verfügen in der Regel über komplexere Gerechtigkeitsvorstellungen als Gewerkschafter „mit Stallgeruch". Eingefahrene Gewerkschaftstraditionen und Handlungsroutinen verfangen bei ihnen nicht. Dafür sind die neuen Aktivisten mit Techniken sozialer Mobilisierung in der Zivilgesellschaft bestens vertraut. Aufgrund ihrer alten Kontakte sehen sie sich zugleich in der Lage, Bündnisbeziehungen zu Nicht-Regierungsorganisationen (NGOs) und Bewegungen außerhalb der Arbeitswelt zu knüpfen, um deren besondere Machtressourcen für die Gewerkschaft zugänglich zu machen (Voss/Sherman, S. 327-331).

Schon die starke Betonung des Bewegungsmoments signalisiert, dass Voss/ Sherman mit dem Organizing-Modell keineswegs ein inhaltloses Set an Rekrutierungstechniken assoziieren. Vielmehr beschreiben sie einen Typus des Gewerkschaftshandelns, der sich durch die Beteiligung von Mitgliedern an der Politikentwicklung, durch unkonventionelle und teilweise höchst konfliktträchtige Aktionsformen sowie eine thematisch breite politische Agenda auszeichnet. Im Zentrum offensiver Organisierungsmodelle steht die Gewinnung neuer Mitglieder. Auch für dieses Ziel werden unkonventionelle, teilweise auch konfrontative Taktiken angewendet (ebd., S. 316). Charakteristisch für entsprechende Ansätze ist die starke Betonung von sozialer Gerechtigkeit und Menschenwürde. So verstanden, impliziert der Übergang zum Organizing-Modell einen strategischen Organisationswandel, der auch die Beziehungen zwischen gewerkschaftlichen
Funktionsträgern und (potenziellen) Mitgliedern nachhaltig verändert. Mittlerweile sind die Befunde von Voss/Sherman durch zahlreiche weitere Studien erhärtet worden (Fiorito 2004; Heery 2005). Nachfolgend beschränken wir uns auf die Präsentation einiger ausgewählter Forschungsergebnisse zu den Inhalten, der organisatorischen Form und den Methoden eines strategischen Organisationswandels von Gewerkschaften.

\subsection{BEWEGUNGSGEWERKSCHAFT}

Die inhaltliche Ausrichtung weit gefasster Organizing-Ansätze wird von einem Teil der wissenschaftlichen Interpreten (z. B. Frege 2000) unter der Bezeichnung „Social Movement Unionism" thematisiert. Damit ist gemeint, dass Gewerkschaften ihre Mobilisierungs- und Konfliktfähigkeit verbessern wollen, indem sie zentrale gesellschaftliche Themen in ihre politische Agenda integrieren. Ursprünglich war die Kategorie „Bewegungsgewerkschaft" auf neue Arbeiterbewegungen und erstarkende Gewerkschaften in einigen Ländern des Südens gemünzt (Brinkmann et al. 2008, S. 45-68). Diese schwach institutionalisierten Gewerkschaften setzen auf Massenmobilisierung, sie verfügen über eine entwickelte Beteiligungskultur in ihrer Mitgliedschaft, organisieren ihre Kämpfe über die Fabrikgrenzen hinaus und machen Bündnisse mit Bewegungen außerhalb der Arbeitssphäre $\mathrm{zu}$ einem konstitutiven Element ihres strategischen Handelns.

Autoren wie Moody (1997) werten dies als Herausbildung eines völlig neuen Gewerkschaftstyps, der sich vornehmlich außerhalb korporativer Einbindungen formiert. Andere Interpreten sprechen dagegen von einer Neuauflage des alten ,political unionism" (Neary 2002). Jenseits dieser Kontroverse thematisieren Forscher, die eine starke Verknüpfung von OrganizingAnsätzen und Bewegungsgewerkschaft konstatieren (Nissen 2003, S. 143ff.), einen wichtigen Punkt. Offenkundig fällt es den Gewerkschaften, die vornehmlich als bes-

\footnotetext{
4 Das Konzept ist selbst in der US-Gewerkschaftsbewegung umstritten. Fortwährende Auseinandersetzungen mündeten 2005 in die Gründung eines eigenständigen Dachverbandes Change to Win (CTW) der "Organizing-Gewerkschaften", der mittlerweile etwa sechs Millionen Mitglieder vertritt.
} 
sere Problemlöser mit dem Management konkurrieren, schwer, organisationspolitisch erfolgreich zu sein (Cregan 2005). Insofern passen Interessenpolitiken, die Gerechtigkeitsfragen gegenüber dem Nachweis wirtschaftlicher Effizienz priorisieren (Aronowitz 2005), in vielen Fällen offenbar besser zum Anspruch offensiver Organizing-Ansätze (Fantasia/Voss 2004, S. 127130) als wertschöpfungsorientierte Strategien. Allerdings sind die Forschungsergebnisse hier nicht eindeutig. Wie im Fall der United Auto Workers (UAW) und ihrer Beteiligung an der Reorganisation von Arbeitsprozessen (Gruppenarbeit, Problemlösungsteams, Kooperation mit dem Management), können Organizing-Ansätze auch im Kontext konsens- und wettbewerbsorientierter Interessenpolitiken erfolgreich sein (Hurd et al. 2003). Auch weil Bewegungspolitiken sich nicht beliebig auf Dauer stellen lassen, ringen nicht nur die südlichen Bewegungs-, sondern auch die nordamerikanischen „Organizing-Gewerkschaften" um eine Institutionalisierung ihrer Verhandlungsmacht. Festhalten lässt sich aber, dass Organizing-Ansätze in vielen Fällen in interessenpolitische Strategien eingebettet sind, die zentrale gesellschaftliche Themen und Konfliktfelder offensiv zu besetzen suchen.

\subsection{BETEILIGUNGSGEWERKSCHAFT}

Direkte Mitgliederbeteiligung ist für viele Autoren eine zentrale politisch-organisatorische Form des Organizing. Fiorito (2004) sieht in der Dezentralisierung von Entscheidungen, in offenen Strukturen, die eine Beteiligung bis hin $\mathrm{zu}$ „Mitgliederselbstbestimmung" erlauben, eine entscheidende Erfolgsbedingung offensiver Organizing-Konzepte. Dabei geht es nicht allein um die Verbreiterung der aktiven Basis einer Gewerkschaft. Die Beziehungen zwischen Aktivisten und „normalen“ Mitgliedern beruhen, wie Beaud/Pialoux (2004) in ihrer großartigen Studie über das Peugeot-Werk in Sochaux gezeigt haben, auf einem eingespielten „System der Erwartungen und Errungenschaften" (ebd., S. 259), das aufgrund von Veränderungen im Arbeitsprozess und in der Zusammensetzung der Belegschaften periodisch erneuert werden muss. Empirische Forschungen haben belegt, dass die zunehmende soziale Heterogenität potenzieller Mitglieder nicht automatisch zu einem Verlust an gewerkschaftlicher Bindekraft führen muss. Viel- mehr wird eine verbesserte Teilhabe der Mitglieder an Organisationsentscheidungen zur entscheidenden Einflussgröße. Innergewerkschaftliche Demokratie und reale Partizipationschancen besitzen laut Lévesque et al. (2005) die mit Abstand höchste Erklärungskraft bei der Abwehr von Mitgliederunzufriedenheit. Markowitz (1999) zeigt darüber hinaus, dass die Stabilisierung partizipativer Beziehungen zwischen Funktionsträgern und neu gewonnenen Mitgliedern wichtig ist, um Gewerkschafter „after organizing“ in der Organisation zu halten. Innerorganisatorische Mitbestimmung gilt Autoren wie Clawson (2005) zudem als Schlüssel für eine erfolgreiche Ansprache gewerkschaftlich bislang schwach repräsentierter Gruppen (Frauen, Prekäre, Minderheiten). Kommunikation, ,von Angesicht zu Angesicht “ und Authentizität der aktiven Gewerkschafter (Vertretung durch Gleiche) sind demnach ausschlaggebend für nachhaltig veränderte Beziehungen zwischen Repräsentanten und Repräsentierten.

Sprechen solche Forschungsergebnisse für einen engen Zusammenhang zwischen Mitgliederpartizipation und organisatorisch-politischer Bindekraft der Gewerkschaften, bleibt doch offen, ob und wie sich ein beteiligungsorientierter Politikstil längerfristig durchhalten lässt. Der Hauptgrund für diese Schwierigkeit wurzelt keineswegs in der von Frege (2000) zu Recht monierten Kluft zwischen partizipativer Rhetorik und der zentralistischen Praxis mancher "Organizing-Gewerkschaften“. Aus der Bewegungsforschung ist bekannt, dass es nicht nur schwer, sondern geradezu unmöglich ist, ein hohes Partizipationsniveau von Mitgliedern über längere Zeiträume hinweg zu stabilisieren. „Partizipationsarbeit" können alte wie neue Mitglieder häufig nur auf Zeit, themen- und projektgebunden leisten; sie wollen dies, wenn es wirklich etwas zu entscheiden gibt, aber nicht, um Beteiligungsrituale zu befriedigen. Insofern werden sich auch die intelligentesten Organizing-Ansätze immer wieder mit dem Problem konfrontiert sehen, dass stärker partizipative und eher repräsentative Ansätze einander abwechseln und ergänzen müssen.

\subsection{KAMPAGNENGEWERKSCHAFT}

Organizing-Ansätze zeichnen sich in der Regel durch einen flexiblen Einsatz unterschiedlicher Taktiken, Instrumente und
Methoden aus. Eine strikte Kampagnenorientierung gilt vielen Autoren als geeignetes Mittel, um Bewegungsorientierung und Mitgliederpartizipation projektgebunden $\mathrm{zu}$ verstetigen. Aus den unterschiedlichen Kampagnenformen ragen die Comprehensive Campaigns heraus. Dabei handelt es sich um einen Kampagnentyp, in dessen Verlauf sich die Gewerkschaften selbst nachhaltig verändern. Bronfenbrenner/Hickey (2004) gelangen in ihrer Analyse zu dem überraschenden Ergebnis, dass positive Resultate nicht so sehr von der Branche oder der Unternehmensstruktur abhängen, sondern vor allem von der Art und Intensität der Kampagne beeinflusst werden. Ein Großteil dessen, was die beiden Autoren als Essentials einer erfolgreichen Kampagnenorientierung benennen (ebd., S. 37-41), wirkt auf den ersten Blick wenig spektakulär. Das gilt etwa für Trainingsmaßnahmen auch während der Kampagne oder die Festlegung von Zwischenzielen. Anderes, wie z. B. die Implementierung von Kampagnen auf dezentraler Ebene, das Vermeiden innergewerkschaftlicher Organisationskonkurrenzen oder die internationale Bearbeitung von Themen in multinationalen Unternehmen, erscheint naheliegend, markiert aber doch einen deutlichen Kontrast zu gewerkschaftlichen Organisationsroutinen.

Zwei Charakteristika implizieren indessen eine besondere Kampagnen-Qualität. Die Beifügung „comprehensive“ bedeutet in der einschlägigen Literatur zunächst, dass Aktionen über die unmittelbaren Kontrahenten hinaus auch auf Aktionäre, Zulieferer, Abnehmer oder Kundengruppen ausgedehnt werden sollen, um so den Druck auf einen Konzern oder auf die Unternehmen einer Branche zu maximieren und ein Höchstmaß an Organisationserfolgen zu erzielen. „Comprehensive" kann aber auch mit „verstehend“ oder „begreifend“ übersetzt werden. Damit ist gemeint, dass erfolgreiche Kampagnen empirische Recherchen und Analysen von Machtverhältnissen voraussetzen, um angemessene Organizing-Ziele identifizieren und eine Schrittfolge eskalierender Taktiken festlegen zu können. Ein solches Vorgehen erfordert eine besondere Qualität der Zusammenarbeit von Gewerkschaftern und Wissenschaftlern, die sich im Team zunächst ein gemeinsames Verständnis einer Branche oder eines Unternehmens samt der anvisierten Beschäftigtengruppen erarbeiten müssen. Dazu bedarf es nicht 
nur eines Sets an angemessenen Forschungsmethoden, sondern auch eines Wissenschaftler-Typus, der bereit ist, sich auf die „Niederungen“ gewerkschaftlicher Basisarbeit einzulassen.

Insgesamt belegen die einschlägigen Studien (Gall 2005), dass eine Kampagnenorientierung höchst Unterschiedliches bedeuten kann. Während sich die internen Hindernisse (fehlende Organizing-Tradition, interner Widerstand, geringe Unterstützung durch die Zentrale) für erfolgreiches Organizing in vielen Ländern gleichen, gibt es bei den externen Hindernissen z. T. erhebliche Unterschiede. In den USA steht eine große Mehrheit der Arbeitgeber gewerkschaftlichen Aktivitäten negativ gegenüber; das ist in Großbritannien teilweise anders (Heery/Simms 2007). Dort sind Organizing-Kampagnen in Betrieben signifikant erfolgreicher, in denen die Arbeitgeber keine explizit gewerkschaftsfeindliche Politik verfolgen. Entscheidend ist aber nicht, ob Kampagnen eher konfrontativ oder stärker kooperativ ausgerichtet sind. Vielmehr sind solche Gewerkschaften besonders erfolgreich, die ein weites Spektrum an Taktiken beherrschen und es optimal auf das jeweilige Feld anzuwenden verstehen.

\section{Organizing - eine strategische Option?}

Organizing, Bewegungsorientierung, Mitgliederpartizipation und Kampagnenfähigkeit bezeichnen einen Möglichkeitsraum, in dem eine strategische Wahl von Gewerkschaften erfolgen kann. Aber ist es aus der Perspektive der DGB-Gewerkschaften überhaupt sinnvoll, sich auf dieses Terrain zu begeben? Carola Frege (2000) hat diese Frage mit einer gehörigen Portion Skepsis beantwortet. Nach ihrer Ansicht bezeichnet Organizing bislang weder ein ausgereiftes Konzept noch einen Ansatz mit eingebauter Erfolgsgarantie. Auch bezweifelt sie die Übertragbarkeit auf deutsche Verhältnisse: So habe Mitgliederwerbung in dem stark verrechtlichten deutschen Arbeitsbeziehungssystem bislang nur eine untergeordnete Rolle gespielt, faktisch sei die Aufgabe Betriebsräten übertragen worden. Wegen ihrer starken institutionellen Einbindung hätten die Gewerkschaften nur selten zum Mittel der Mitglie- dermobilisierung greifen müssen. Zwar könne die Mitgliederkrise DGB-Gewerkschaften zur Adaption einzelner Praktiken motivieren, eine Übernahme des gesamten Organizing-Konzepts oder gar ein Wiederaufleben von Gewerkschaften als soziale Bewegungen sei aber unwahrscheinlich.

Ohne Zweifel trifft Frege damit wichtige Punke. Das gilt umso mehr, als die strategische Positionierung der DGB-Gewerkschaften noch immer eine andere ist als die der nordamerikanischen „Organizing-Gewerkschaften“. In den USA entspringt die starke Betonung von Bewegungsorientierung und Coalition Building mit NGOs und Graswurzelinitiativen hauptsächlich der gewerkschaftlichen Schwäche in Betrieben und Unternehmen, sprich: der minimierten institutionellen Verhandlungsmacht. Aus diesem Grund macht es Sinn, die Durchsetzungsfähigkeit der Gewerkschaften mittels unkonventioneller Bündnispolitik erhöhen zu wollen. In Deutschland sind die Verhältnisse - noch - anders. Weder ist die Erosion institutioneller Verhandlungsmacht so weit fortgeschritten wie in den angelsächsischen Kapitalismen, noch sind soziale Bewegungen, NGOs und Graswurzelinitiativen derart stark, dass Bündnisse mit ihnen schwindende gewerkschaftliche Organisationsmacht kompensieren könnten. Auch aus diesem Grund scheuen die Gewerkschaften mehrheitlich davor zurück, Verhandlungsmacht, wie sie Mitbestimmung und Tarifsystem noch immer bieten, zugunsten einer riskanten Bündnispolitik mit oppositionellen Bewegungen zu opfern.

\subsection{DIE ORGANISATIONSPOLITISCHE FALLE}

Dies in Rechnung gestellt, gibt es dennoch genügend Gründe für rasches Organisationslernen. So sorgt der - von Frege unterschätzte - Druck auf die institutionalisierte Verhandlungsmacht der Gewerkschaften für einen Bedeutungsgewinn der strukturellen Macht privilegierter Berufsgruppen. Angesichts einer Konkurrenz, die auch mittels Prekarisierung von Beschäftigungsverhältnissen ausgetragen wird, tendieren Gruppen mit hoher Primärmacht wie die Lokführer oder die Ärzte dazu, ihre Interessen separat von anderen Beschäftigtengruppen wahrzunehmen. Anders als unternehmenskonforme, „gelbe“ Gewerkschaften zeichnen sich ihre nach absoluten Mitgliederzahlen eigentlich schwachen Or- ganisationen durch hohe Lohn- und Gehaltsforderungen sowie hart geführte Konflikte aus. Die Erosion institutioneller ( Tarif-)Macht der großen Mitgliedsgewerkschaften produziert so ein Revival separater Interessenkämpfe strukturell mächtiger Berufsgruppen. Parallel dazu wächst aber auch die Ungleichheit unter den Lohnabhängigen, weil vertretungsschwache Gruppen wie z. B. die $18 \%$ Niedriglohnbezieher in Vollzeitbeschäftigung (Bosch/Weinkopf 2007) in dieser Konfliktkonstellation tendenziell den Kürzeren ziehen. Damit befinden sich die DGB-Gewerkschaften in einer Art Zwickmühle: Bleiben sie in der Fläche weniger durchsetzungsfähig, weil die alten korporativen Mechanismen nicht mehr funktionieren, drohen sie zwischen - teilweise schwach organisierten - Berufsgruppen mit großer struktureller Macht und dem wachsenden Heer prekär Beschäftigter eingeklemmt zu werden und weiter an Einfluss zu verlieren. Diese Konstellation ist es, die die Frage nach strategischen Alternativen aufwirft und das Interesse an Organizing-Praktiken weckt.

\subsection{SCHRITTE ZUR KAMPAGNENORIENTIERUNG}

Tatsächlich haben Lernprozesse längst begonnen, und sie machen sich auch in Organisationsbereichen bemerkbar, in denen eine Orientierung an „Organizing-Ansätzen" nicht vorhanden ist oder gar abgelehnt wird. Relativ unkompliziert erscheint auf den ersten Blick die Übernahme einzelner Instrumente des Organizing. So orientiert sich die LIDL-Kampagne von ver.di explizit am Vorbild einer Comprehensive Campaign (Schreieder 2007). Anders als im Falle der US-Gewerkschaften zielt die Kampagne jedoch zunächst auf Betriebsratsgründungen. Von der Etablierung betrieblicher Interessenvertreter erhofft sich die Gewerkschaft, allmählich auch die Beitrittsschwelle für die Beschäftigten des Unternehmens senken zu können. Im Vergleich zu den US-amerikanischen Vorbildern mangelt es der Kampagne allerdings erheblich an Ressourcen und an organisatorischer Verbindlichkeit.

Ein anderes Beispiel sind Kampagnen, die auf eine verbesserte Repräsentation und eine Rekrutierung prekär Beschäftigter zielen. Der inzwischen von mehreren Mitgliedsgewerkschaften getragenen Mindestlohn-Kampagne kommt hier eine symbolische Funktion zu. Nicht minder wichtig 
sind dezentrale Initiativen. So haben zahlreiche DGB-Gliederungen (z. B. Oldenburg) mit Bestandsaufnahmen zur Entwicklung prekärer Beschäftigung begonnen. Im Organisationsbereich der IGM gibt es inzwischen mehrere Leiharbeitsprojekte. Der Berliner IGM-Bezirk hat mit einem „Sozialreport Zeitarbeit" für öffentliche Reaktionen gesorgt. Weil der Leiharbeitstarif zwar eine „Haltelinie nach unten “ darstellt, in manchen Branchen aber den Abstand $\mathrm{zu}$ Tariflöhnen zementiert, sind IGM-Gliederungen dazu übergegangen, in den Einsatzbetrieben von Leiharbeitern gleiches Geld für gleiche Arbeit zu verlangen. Dabei werden sie von den Betriebsräten großer Unternehmen (z. B. ThyssenKrupp) unterstützt. Noch fehlt es an geeigneten Beratungs- und Organisationsstrukturen für potenzielle Mitglieder, die räumlich verstreut arbeiten und häufig den Einsatzort wechseln. Dennoch beinhaltet dieser Ansatz ein Potenzial, das sich - weit über das Leiharbeitsthema hinaus - zu einer Antidiskriminierungspolitik in Betrieben und Unternehmen ausbauen ließe. Interessant ist, dass punktuelle Organisierungserfolge dort möglich werden, wo, wie z. B. in der Verwaltungsstelle Ingolstadt, die gewerkschaftlichen Protagonisten auf Formen der Selbstorganisation von Leiharbeitern setzen.

\subsection{DIE ENTDECKUNG DER MITGLIEDER}

Damit ist bereits die politisch-organisatorische Form des Organizing, die Mitgliederpartizipation, berührt. Hier vollziehen sich im „Kerngeschäft“ der deutschen Gewerkschaften, in der Betriebs- und Tarifpolitik, erstaunliche Veränderungen. Gemeint ist die Neustrukturierung der Beziehungen von Betriebsräten, gewerkschaftlichen Funktionsträgern und (potenziellen) Mitgliedern, wie sie im Organisationsbereich der IG Metall teilweise erprobt wird. Angesichts der Legitimationsverluste, die betriebliche Konzessionspolitiken seit einiger Zeit verzeichnen (Rehder 2006), sind einzelne Bezirke und auch lokale Gliederungen der IG Metall dazu übergegangen, Standortvereinbarungen an Voten von Gewerkschaftsmitgliedern oder Belegschaften zu knüpfen. Teilweise werden Quoren vereinbart oder, wie im Bezirk Küste, betriebliche Tarifkommissionen eingesetzt. Selbst wenn direkte Partizipation niedrigschwellig, also auf dem Niveau von Belegschafts- befragungen oder einer Beteiligung von Mitgliedern an der Aufstellung von Betriebsratslisten, erfolgt, verändert sie die betrieblichen Machtspiele erheblich. Verhandlungen sind dann nicht mehr ausschließlich die Sache von Geschäftsleitungen, Betriebsräten und Gewerkschaftssekretären. Stattdessen tritt mit den Gewerkschaftsmitgliedern ein neuer Akteur in Erscheinung, dessen Beteiligung an substanziellen Entscheidungen Ungewissheiten für die etablierten Betriebs- und Tarifparteien mit sich bringt. Einmal nach ihrer Meinung gefragt, werden sich Gewerkschaftsmitglieder und Belegschaften derartige Beteiligungsmöglichkeiten wohl auch künftig nicht mehr ohne Weiteres nehmen lassen. Die Besser-statt-billiger-Kampagne des IGM-Bezirks NRW ist ein exemplarischer Versuch, solche Ansätze zu bündeln und sie auf das Innovationsthema auszudehnen. Dabei geht es nicht nur um die qualitative Verbesserung von Standortvereinbarungen (Festlegung von Innovationszielen). Vielmehr sollen betriebliche Verhandlungen an einen direkten organisationspolitischen Nutzen der Gewerkschaften gekoppelt werden. Über die betriebs- und tarifpolitischen Zielsetzungen hinaus sind sie wesentlich auf das Ziel einer Stärkung gewerkschaftlicher Organisationsmacht ausgerichtet.

Noch scheint die Prognose, dass „betriebliche Interessenvertretungen nach einer Phase des kooperativen Co-Managements nun vermehrt in eine basisorientierte Interessenvertretung hineinsteuern" (Rehder 2006, S. 242), einigermaßen verwegen. Denn der Verbreitungsgrad unkonventioneller Mitgliederpartizipation ist vorerst wohl noch gering. Auch ist die Initiierung dezentraler Beteiligungsformen innergewerkschaftlich höchst umstritten, ihre Auswirkungen auf überbetriebliche Tarifabkommen lassen sich noch nicht präzise benennen, und teilweise fehlt es schlicht an partizipationsbereiten Gewerkschaftsmitgliedern. Dennoch kann kein Zweifel bestehen, dass - ganz im Sinne des Organizing-Modells - das Problem einer Stärkung gewerkschaftlicher Organisationsmacht innergewerkschaftlich, wie auch in der betrieblichen Arena, wieder an Bedeutung gewinnt.

\subsection{INHALTE}

Selbst beim schwierigen Thema „Gewerkschaften und soziale Bewegungen“ lassen sich bei näherem Hinsehen größere Schnittmengen feststellen, als man auf den ersten Blick vermuten könnte. Zwar haben die Anti-G8-Mobilisierungen für die DGBGewerkschaften eine ungleich geringere Bedeutung als für einen Teil der „Organizing-Unions" in den USA; die Themen der globalisierungskritischen Bewegungen sind aber auch für die deutschen Gewerkschaften relevant. Es gibt einen - teils formalisierten (Verbindungsbüro der IGM zu den sozialen Bewegungen), teils informellen - Austausch mit sozialen Bewegungen auf Spitzenebene wie an der Basis. Einige Gewerkschaften sind mit Repräsentanten an der Sozialforen-Bewegung beteiligt, teilweise kommt es zu einem inhaltlich-politischen (Arbeitsmarktpolitik, Bahn-Privatisierung) und in begrenztem Maße auch personellen Austausch.

In diesem Zusammenhang darf nicht übersehen werden, dass die deutschen Gewerkschaften den amerikanischen „Organizing-Unions" auf vielen betriebspolitischen Feldern ein gutes Stück voraus sind. Das gilt z. B. für arbeitspolitische Gestaltungsansätze, wie sie im IGM-Projekt „Gute Arbeit" zu unterschiedlichen Themen (Gesundheitsprävention, demografischer Wandel, Leistungssteuerung) erprobt werden. Diese Ansätze sind auch geeignet, in den expandierenden Segmenten mit qualifizierten, teilweise managementnahen Tätigkeiten Beachtung zu finden. Bislang befinden sie sich aber noch im Stadium der Erprobung, und sie sind noch nicht systematisch mit Initiativen zur Stärkung gewerkschaftlicher Organisationsmacht verbunden.

\section{Fazit}

Natürlich sind dies, am Maßstab einer Überwindung der gewerkschaftlichen Repräsentationskrise gemessen, allenfalls zarte Pflänzchen der Erneuerung, deren Wirkungen erst mit gebührendem zeitlichen Abstand bewertet werden können. Bislang fehlt es zudem an gesicherten wissenschaftlichen Erkenntnissen über die Resultate innovativer gewerkschaftlicher Praktiken. Als Besonderheit gegenüber der nordamerikanischen Kontrastfolie springt ins Auge, dass zahlreiche Aktivitäten auf eine Sensibilisierung vorhandener Betriebsräte oder eine Neugründung von Interessen- 
vertretungen zielen. Auch aus diesem Grund schlagen sich Kampagnen und Initiativen nicht unmittelbar in Mitgliederzuwächsen nieder. Allerdings zeigt sich ebenfalls, dass Gewerkschaftsgliederungen sich durchaus als Schrittmacher einer aktiven Stärkung ihrer Organisationsmacht betätigen können. Bis zu einer systematischen Ausrichtung des Funktionärskörpers auf offensives Organizing („Mitglieder werben Mitglieder") ist es aber noch ein weiter Weg. Daher fällt ein vorläufiges Fa- zit notgedrungen zwiespältig aus. Trotz mancher Gemeinsamkeiten mit Organizing-Ansätzen haben sich innovative Praktiken bislang nicht zu einer kohärenten Interessenpolitik verdichten können. Es fehlt an einer geeigneten organisationspolitischen Infrastruktur, an Organizing-Akademien, spezifischen Qualifizierungsangeboten, aber auch an geeigneten Kooperationsstrukturen mit Wissenschaftlern, Journalisten und Medienfachleuten. Und auch Funktionsträger, die bereit und in der
Lage sind, ihren Aktivitätsschwerpunkt jenseits der Organisationsroutinen zu setzen, sind noch rar gesät. Von einem wirklichen ,strategischen Organisationswandel“, wie ihn die Organizing-Debatte thematisiert, kann daher in den DGB-Gewerkschaften vorerst noch keine Rede sein. Punktuell sind die Praktiker bei der Rekonstruktion gewerkschaftlicher Organisationsmacht in Betrieben aber schon weiter, als es der grassierende Niedergangsfatalismus Glauben machen will.

\section{ITERATUR}

Aronowitz, S. (2005): On the Future of American Labor, in: Working USA. The Journal of Labor and Society 3, S. 271-291

Beaud, S./Pialoux, M. (2004): Die verlorene Zukunft der Arbeiter, Kons$\tan z$

Bosch, G./Weinkopf, C. (Hrsg.) (2007): Arbeiten für weniger Geld. Niedriglohnbeschäftigung in Deutschland, Frankfurt/M.

Brinkmann, U./Choi, H./Detje, R./Dörre, K./Holst, H./Karakayali, S./Schmalstieg, C. (2008): Strategic Unionism. Aus der Krise zur Erneuerung?, Wiesbaden

Bronfenbrenner, K./Hickey, R. (2004): Changing to Organize: A National Assessment of Union Organizing Strategies, in: Milkman, R./Voss, K. (Hrsg.): Rebuilding Labor. Organizing and Organizers in the New Union Movement, Ithaca, S. 17-61

Clawson, D. (2005): Organizing, Movements and Social Capital, in: Labor Studies Journal 4, S. 37-44

Cregan, C. (2005): Can organizing work? An inductive analysis of individual attitudes toward union membership, in: Industrial and Labor Relations Review 2, S. 282-304

Dörre, K./Röttger, B. (2006): Im Schatten der Globalisierung. Strukturpolitik, Netzwerke und Gewerkschaften in altindustriellen Regionen, Wiesbaden

Dribbusch, H. (2003): Gewerkschaftliche Mitgliedergewinnung im Dienstleistungssektor. Ein Drei-Länder-Vergleich im Einzelhandel, Berlin Dribbusch, H. (2007): Das Organizing-Modell. Entwicklung, Varianten, Umsetzung, in: Bremme, P./Fürniß, U./Meinecke, U. (Hrsg.): Never work alone, Organizing - ein Zukunftsmodell für Gewerkschaften, Hamburg, S. $24-52$
Fantasia, R./Voss, K. (2004): Hard Work. Remaking the American Labor Movement, Berkeley

Fiorito, J. (2004): Union Renewal and the Organizing Model in the United Kingdom, in: Labor Studies Journal 2, S. 21-53

Fligstein, N. (2001): The Architecture of Markets. An Economic Sociology of Twenty-First- Century Capitalist Societies, Princeton

Frege, C. M. (2000): Gewerkschaftsreformen in den USA. Eine kritische Analyse des "Organisierungsmodells", in: Industrielle Beziehungen 3, S. $260-280$

Frege, C. M./Kelly, J. (Hrsg.) (2004): Varieties of Unionism. Strategies for Union Revitalization in a Globalizing Economy, Oxford

Gall, G. (2005): Organizing Non-Union Workers as Trade Unionists in the 'New Economy' in Britain, in: Economic and Industrial Democracy 1, S. 41-63

Geiselberger, H. (2007): Social Movement Unionism. Tomaten des Zorns, in: Geiselberger, H. (Hrsg.): Und Jetzt? Politik, Protest und Propaganda, Frankfurt/M

Harvey, D. (2006): Limits to Capital, London

Heery, E./Simms, M. (2007): Employer Responses to Union organising in the United Kingdom, Manuskript, Warwick

Heery, E. (2005): Sources of change in trade unions, in: Work, Employment \& Society 1, S. 91-106

Huber, B./Burkhard, O./Wagner, H. (Hrsg.) (2006): Perspektiven der Tarifpolitik. Im Spannungsfeld von Fläche und Betrieb, Hamburg Hurd, R./Milkman, R./Turner, L. (2003): Reviving the American Labour Movement. Institutions and Mobilization, in: European Journal of Industrial Relations 1, S. 99-117 
Lévesque, C./Murray, G./Le Queux, S. (2005): Union Disaffection and Social Identity. Democracy as a Source of Union Revitalization, in: Work and Occupations 4, S. 400-422

Markowitz, L. (1999): Worker Activism after Successful Union Organizing, Armonk N. Y.

Milkman, R./Voss, K. (Hrsg.) (2004): Rebuilding Labor. Organizing and Organizers in the New Union Movement, Ithaca

Moody, K. (1997): Workers in a Lean World, London

Neary, M. (2002): Labour Moves. A Critique of the Concept of Social Movement Unionism, in: Dinerstein, A. C./Neary, M. (Hrsg.): The Labour Debate. An investigation into the theory and reality of capitalist work, Aldershot, S. 149-178

Nissen, B. (2003): Alternative Strategic Directions for the U. S. Labor Movement. Recent Scholarship, in: Labor Studies Journal 1, S. 133-155
Rehder, B. (2006): Legitimitätsdefizite des Co-Management. Betriebliche Bündnisse für Arbeit als Konfliktfeld zwischen Arbeitnehmern und betrieblicher Interessenvertretung, in: Zeitschrift für Soziologie 3, S. 227242

Schreieder, A. (2007): Die LIDL-Kampagne - ein Zukunftsmodell der Gewerkschaften, in: Bremme, P./Fürniß, U./Meinecke, U. (Hrsg.): Never work alone, Organizing - ein Zukunftsmodell für Gewerkschaften, Hamburg, S. 153-174

Silver, B. J. (2005): Forces of Labour. Arbeiterbewegung und Globalisierung seit 1870, Berlin

Voss, K./Sherman, R. (2000): Breaking the Iron Law of Oligarchy. Union Revitalization in the American Labor Movement, in: American Journal of Sociology 2, S. 303-349

Wright, E. O. (2000): Working Class Power, Capitalist Class Interests, and Class Compromise, in: American Journal of Sociology 4, S. 957-1002 